\title{
Denoising of 3D magnetic resonance images using non-local PCA and Transform-Domain Filter
}

\author{
Laraib Kanwal, \\ Muhammad Usman Shahid
}

\begin{abstract}
The Magnetic Resonance Imaging (MRI) technology used in clinical diagnosis demands high Peak Signal-to-Noise ratio (PSNR) and improved resolution for accurate analysis and treatment monitoring. However, MRI data is often corrupted by random noise which degrades the quality of Magnetic Resonance (MR) images. Denoising is a paramount challenge as removing noise causes reduction in the fine details of MRI images. We have developed a novel algorithm which employs Principal Component Analysis (PCA) decomposition and Wiener filtering. We have proposed a two stage approach. In first stage, non-local PCA thresholding is applied on noisy image and second stage uses Wiener filter over this filtered image. Our algorithm is implemented using MATLAB and performance is measured via PSNR. The proposed approach has also been compared with related state-of-art methods. Moreover, we present both qualitative and quantitative results which prove that proposed algorithm gives superior denoising performance.
\end{abstract}

Keywords-MRI, PCA, Denoising, BM4D, PRI-NL-PCA, Wiener filter.

\section{INTRODUCTION}

Magnetic Resonance Imaging (MRI) is three-dimensional imaging technique extensively used in clinical diagnosis. It is superior to other diagnostic modalities due to its ability to easily differentiate and highlight tissues. MRI scanners form an image with the help of

Laraib Kanwal, Muhammad Usman Shahid

Electrical Engineering Department

National University of Computer \& Emerging Sciences

Lahore, Pakistan 
magnetic field and radio waves. When human body is placed in strong magnetic field (1.5 Tesla) provided by MRI device then radio frequency pulses fall incident on the target area. As a result, waves are reflected and absorbed. The reflected waves are detected and 3D image in formed on scanner.

The accuracy of MRI images is seriously degraded by random noise during acquisition, transmission and storage. As a result, the reliability of important tasks (registration, segmentation, visualization) decreases. The major noise in MRI is thermal noise (also known as Johnson noise). Generally, there are two approaches to reduce noise and improve SNR of MRI images. One way is to average the acquired data multiple times. However, this method increases the acquisition time whereas medical images demand quick response. Sometimes MRI scan process can go from 20 minutes to many hours. Long acquisition time results in increased electricity and time cost as well.

There are various methods for MRI denoising. They can be classified in two broad categories that are filtering and transform domain. NonLocal Means (NLM) filter proposed by Buades et al, performs best in filtering domain while contourlet transform gives good denoising performance in transform domain [1].

The reminder of the paper is organized as follows. In Section II we present the literature review of all denoising algorithms. Section III describes the proposed method and its implementation. The experimental results on phantom dataset are analyzed in Section IV. Finally, Section V concludes the paper.

\section{LITERATURE REVIEW}

A lot of work has been done for denoising MRI images. Many variations of NLM algorithm have been proposed. Manjon et al, proposed a modification of NLM algorithm for denoising multispectral MRI images. This method is termed as Multispectral NLM (MNLM) algorithm. Most of the MRI denoising methods are applied on single channel images ignoring the multispectral nature of these images. In multispectral MRI images, information of many channels are combined [2]. This paper focuses on noise reduction and SNR improvement in multispectral MRI images. MNLM algorithm is less 
efficient for noise removing but iterations can be increased for good results.

Coupe et al. [3] proposed multithreading approach to overcome the main drawback of the NLM algorithm. For 3D MRI data, the computation of NLM algorithm takes significant amount of time so computational burden increases. Hence, optimized version of NLM filter reduces computational time by factor of 50 using parallelized and optimized implementation. Later Coupe et al, extended the work by further reducing the computational time by the factor of 60 . This is achieved by optimized pre-selection of voxel along with block-wise implementation [4]. Coupe et al, further continued the work by introducing wavelet sub-bands mixing (WSM) in fully automatic 3D optimized block-wise NLM filter. In this technique, the image quality and computational time are being enhanced. NLM filter combined with wavelet decomposition outperforms the optimized block-wise NLM implementation [5].

Gal et al, proposed an algorithm called Dynamic Nonlocal Means (DNLM) for removing redundancy (time axis) [6]. 


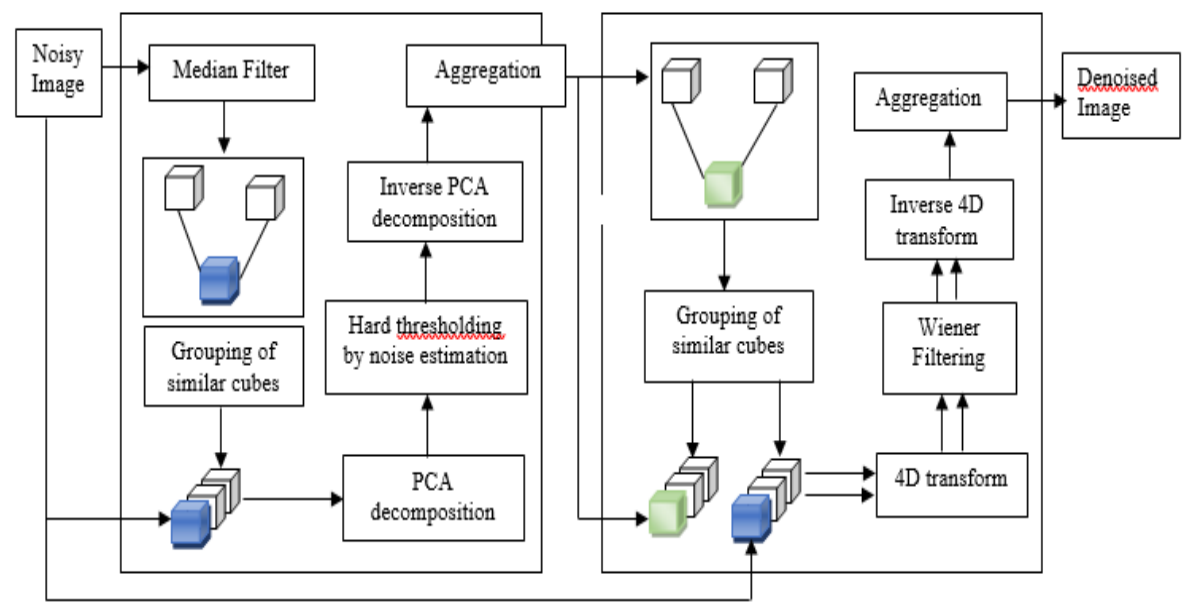

Figure 1. Block Diagram of Proposed Method

DNLM algorithm makes use of information redundancy in data volume acquired at different time intervals. DNLM algorithm achieves good denoising performance visually. Moreover, it has fast execution time as compared to previously proposed methods. Liu et al, proposed Preprocessed Enhanced NLM filter (PENLM) for 3D MR images. In this filter, the image is first pre-processed with Gaussian filter then NLM filter is applied to squared magnitude image. The introduction of Gaussian filter before NLM filter reduces the noise disturbance [7].

$\mathrm{Hu}$ et al, used combination of NLM filter with the Discrete Cosine Transform (DCT). In this method, DCT transforms the image from time domain to frequency domain. DCT has promising properties of excellent energy compaction and dimensionality reduction which is used to suppress noise. Then NLM filter is applied and similarity 
weights are calculated to this lower- dimensional sub-space of DCT. The traditional NLM filter computes similarity weights in gray level information rather than DCT sub-space. As a result, the proposed filter gives better denoising performance [8].

Manjon et al, proposed a new approach for an efficient 3D MRI denoising. It is based on two important properties of MR images that is sparseness and self-similarity. The 3D MRI image is initially prefiltered by DCT, followed by hard thresholding then NLM filter is applied. So, Pre-filtered NLM 3D (PRI- NLM3D) algorithm is proposed [9]. Alessandro presented Block Matching 4D (BM4D) algorithm which implements grouping and collaborative filtering. Similar cubes are grouped together in a stack and filtered in transform domain. The 4-D transform applied on the similar groups exploit local as well as non-local correlation present among voxels of each cube and between corresponding voxels of different cubes. BM4D is composed of two cascaded stages: Hard-thresholding stage and Wiener-filtering stage. First filters the noisy image using hard-thresholding and second uses collaborative wiener filtering for coefficient shrinkage. The algorithm removes both Gaussian and Rician Noise [10].

José V. Manjón proposed a method that uses lower dimensional subspace. This method have two stages: Non-local Principal Component Analysis (PCA) thresholding and NLM filtering. The noisy image is first pre-filtered by median filter then subsequent steps include: grouping, PCA decomposition, hard thresholding and aggregation. For PCA thresholding strategy, the local noise level is automatically estimated in the image. Then this filtered image is passed to second stage which comprises of rotational invariant NLM filter [11]. This method has been compared with related state-of-art algorithms and shows competitive results.

\section{PROPOSED METHOD}

In the proposed method, similar cubes are grouped together prior performing median filtering. A simple 3D median filter improves the grouping process and provides sparser representation for denoising. PCA decomposition is performed on every created group of similar cubes. Eigenvectors obtained as a result of PCA decomposition are then 
hard thresholded to remove less significant components. Finally, all estimates are combined together using uniform averaging rule and denoised image is obtained. This method is referred as Pri-Non Local PCA (PRI-NL-PCA). The denoised image obtained from PRI-NL-PCA is again passed through grouping phase. Then $4 \mathrm{D}$ transform is applied on groups and Wiener filter is used as collaborative filter. After inverse 4D transform, all estimates are aggregated to get final denoised image. Figure 1 shows the block diagram of proposed algorithm including two stages.

\subsection{PRI-NL-PCA stage}

This stage consists of three steps: grouping, PCA decomposition, thresholding. Firstly, the noisy image is pre-filtered using median filter to get filtered image. By pre-filtering the noisy image prior grouping, we get homogeneous group instead of directly grouping the similar patches. As a result, sparser representations will enable better noise reduction. Coupé has shown that simple 3D median filter greatly improves the group selection process especially for medium and high noise level. Median filter is used for pre-filtering because it can be applied at any point of image irrespective of local noise level. Moreover, it is very efficient for removing noise [12].

\subsection{Non-local PCA denoising}

After pre-filtering, groups of similar cubes are formed by sliding 3D window. For each reference cube, the window slides within search volume to find most similar cubes to the current reference cube. For each point $\mathrm{x}$ of the image, all possible similar cubes are arranged as a row vector in matrix $X$. The similarity between two cubes is measured via Euclidean distance which shows the sum of squared differences between corresponding intensities of two cubes. We consider two cubes similar if their distance is smaller than or equal to threshold $\mathrm{T}_{\text {match }}$. Then similar patches are reordered in a matrix $\mathrm{X}$ whose $\mathrm{N}$ rows correspond to number of grouped patches and $\mathrm{K}$ columns correspond to number of voxels of each 3D patch. Hence $\mathrm{X}$ is an $\mathrm{N} x \mathrm{~K}$ matrix.

PCA decomposition is then performed which uses lower dimensional subspace and shows increased accuracy. PCA is a statistical approach which transforms the correlated variables into uncorrelated principal 
components. Due to this de-correlation property, PCA is used for image denoising. Most of the image details are preserved in first few principal components which are significant while noise is dominated in later components. Therefore, noise can be removed easily. As a result of PCA decomposition, we get eigenvectors and thresholding is applied to remove less significant noise related components. The eigenvectors with standard deviation less than threshold $\mathrm{T}_{P C A}$ are set to zero. Then we invert PCA decomposition and all estimates are combined to get denoised image.

\subsection{PCA based Noise Estimation}

The threshold $T_{P C A}$ is set according to noise level present in image. The correct noise level estimation is necessary to select signal related components and for optimal performance. In order to obtain robust noise level estimator there are two possible cases. If the set of cubes are extracted from homogeneous area, then both mean and median of eigenvalues are expected to be close to noise variance. In another case, if cubes belong to textured areas or edges where signal related components are significant, the mean of eigenvalues will overestimate the variance of noise. This is because of contamination of noise variance by signal variance. However, the median of eigenvalues still will be close to noise variance. Hence, median of eigenvalues is used for noise estimation instead of mean.

The median of eigenvalues given in Eq. 1 is related to noise variance. So, local noise standard deviation can be calculated from median of eigenvalues $\lambda$ as done in [11]

$$
\mathrm{o}^{*}=\beta \sqrt{\operatorname{median}(\lambda)}
$$

where $B$ is correction factor that is ratio between number of selected patches $\mathrm{N}$ and number of voxels of each patch $\mathrm{K}$. Its value is set to 1.16 which is experimentally obtained for $\mathrm{N}=\mathrm{K}$. 
This approach for noise estimation holds good for medium and high noise. However, for low noise conditions in strong edges and textured areas (low group sparseness), this method slightly overestimates the noise level. This overestimation is because the variance of signal can be no more neglected as compared to variance of noise. To reduce the effect of signal in noise estimation, we use a subset of eigenvalues in which first eigenvalues dominated by signal are removed. So, median estimation is performed on this subset to estimate noise. In order to implement this, the eigenvalues whose standard deviation is higher than two times the standard deviation of median of full set of eigenvalues are removed. This new subset is named as trimmed subset of eigenvalues and noise standard deviation is estimated as square root of median of trimmed subset with correction factor $\beta=1.29$ for new subset of eigenvalues [12]. Equation 2 defines it as

$\sigma=\beta \sqrt{\operatorname{median}\left(\lambda_{t}\right)}, \lambda_{t}=\left\{\lambda_{i} \mid \sqrt{\lambda_{i}}<2 \operatorname{median}(\sqrt{\lambda})\right\}$

\subsection{Wiener Filtering stage}

Let suppose $y^{p r i}$ is the denoised image we get after first stage. This image obtained from PRI-NL-PCA stage is passed to the second stage. Again grouping of similar cubes is done but here the noise level of the input image is low as compared to the noisy image. As a result, we expect superior denoising performance because of high sparsification of groups. The cubes of voxels are stacked in 4D groups. Three dimensions are reserved for cubes and fourth dimension shows the direction in which cubes are stacked.

In Wiener filtering step four 1D de-correlation linear transform is applied separately in each dimensions. At first, the group $G^{y^{p r i}}$ is formed from the partly denoised image then Eq.3 defines coefficients of empirical Wiener filter [11] as 


$$
\mathbf{W}=\frac{\left|T_{4 D}^{w i e}\left(G^{y p r i}\right)\right|^{2}}{\left|T_{4 D}^{w i e}\left(G^{y^{p r i}}\right)\right|^{2}+o^{2}}
$$

where $T_{4 D}^{\text {wie }}$ is $4 \mathrm{D}$ transform operator which includes four 1D linear transformation. Similarly, we define another group $\boldsymbol{G}^{\mathbf{Z}}$ extracted from noisy image $z$ using same set of coordinates of similar cubes as used in Wiener filtering stage. So, groups $\boldsymbol{G}^{\boldsymbol{y}^{\boldsymbol{p r i}}}$ and $\boldsymbol{G}^{\boldsymbol{z}}$ have same similar cubes but one group has partly denoised cubes and other has noisy cubes. Then spectrum of noisy group $\boldsymbol{G}^{z}$ is obtained by 4D transform operator as $T_{4 D}^{w i e}\left(G^{z}\right)$. Finally, coefficents of Wiener filter and spectrum of noisy group are multiplied element-by-element to implement coefficient shrinkage as W. $T_{4 \boldsymbol{D}}^{\text {wie }}\left(\boldsymbol{G}^{\boldsymbol{z}}\right)$. The shrunk spectrum is passed through inverse $4 \mathrm{D}$ transform $T_{4 D}^{w i e^{-1}}$ to produce estimate of finally denoised group $\boldsymbol{G}^{\boldsymbol{y}}$ [11]. Equation 4 defines this group as

$$
G^{y}=\tau_{4 D}^{w i e^{-1}}\left(\mathrm{~W} \cdot T_{4 D}^{w i e}\left(G^{z}\right)\right)
$$

At the end, final estimate is obtained by aggregation of group estimate $G^{y}$.

\section{EXPERIMENTAL RESULTS}

Our main objective is to compare the denoising performance of proposed algorithm with state-of-art algorithms. So, we have implemented three existing denoising algorithms: BM4D [11], PRINL-PCA [12] and Proposed method. The performance is measured using PSNR. The parameters of proposed method are adjusted for optimal performance. The four important parameters are cube size $L$, search cube size $N_{s}$, step size $N_{\text {step }}$ the sliding step to process every next reference cube, threshold $T_{\text {match }}$ for grouping of similar cubes and threshold $T_{P C A}$ applied to eigenvalues of PCA decomposition. Table I shows the parameter settings for proposed method. 
Denoising of 3D magnetic resonance images using non-local PCA and Transform-Domain Filter

TABLE 1. PARAMETER SETTINGS FOR THE PROPOSEDALGORITHM

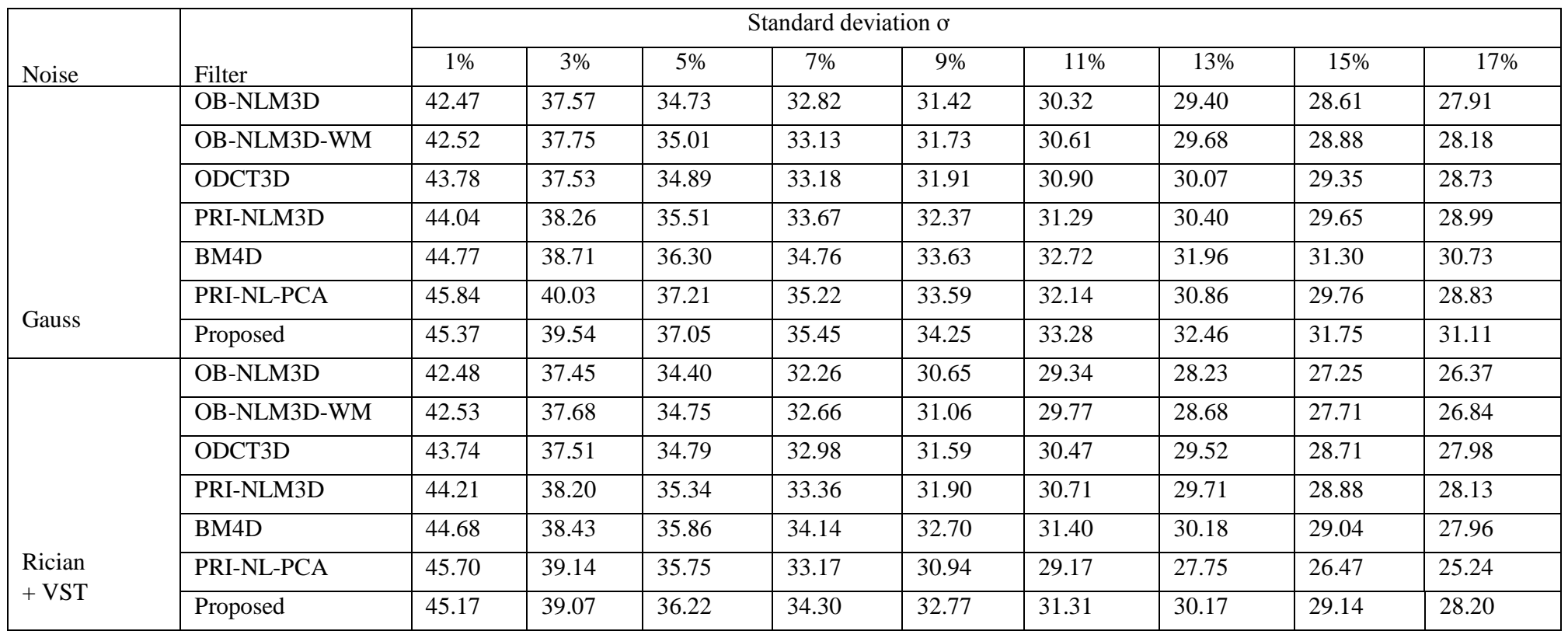


TABLE 2 DENOISING PERFORMANCE OF PROPOSED ALGORITHM

\begin{tabular}{|l|c|c|}
\hline \multicolumn{2}{|l|}{ Parameter } & $\begin{array}{l}\text { Set } \\
\text { values }\end{array}$ \\
\hline Cube size & $\mathrm{L}$ & $4 \times 4 \times 4$ \\
\hline $\begin{array}{l}\text { Search cube } \\
\text { size }\end{array}$ & $N_{s}$ & $7 \times 7 \times 7$ \\
\hline Step size & $N_{\text {step }}$ & 3 \\
\hline $\begin{array}{l}\text { Similarity } \\
\text { threshold }\end{array}$ & $T_{\text {match }}$ & 0.1 \\
\hline PCA threshold & $T_{p c a}$ & $2.1 \%$ \\
\hline
\end{tabular}

\subsection{Simulation Setup}

The simulation results for $\mathrm{T} 1$ weighted modality with different values of noise standard deviation are presented in Table II. We have compared the denoising performance of Proposed algorithm against block matching 4D BM4D [10], pre-filtered non-local PCA PRI-NL-PCA [11], the optimized block-wise non-local means OB-NLM3D [4], optimized block-wise nonlocal means with wavelet mixing OB-NLM3D-WM [5], the oracle-based 3-D DCT ODCT3D [8], and the pre-filtered rotationally invariant nonlocal means PRI-NLM3D [10]. Hence, the proposed algorithm achieves best denoising performance for high noise level giving PSNR improvement of almost $1 \mathrm{~dB}$.

The MR images are available on Open Access Series of Imaging Studies (OASIS) database [13]. The 3D volumes of images are $181 \times 217$ x 181 voxels with voxel resolution of $1 \mathrm{~mm}^{3}$. Moreover, any noise level can be introduced for experiments. The noise standard deviation ranging from $1 \%$ to $17 \%$ is added. The MRI images are corrupted with both Gaussian and Rician noise. Gaussian noise is additive noise while Rician noise is generated by adding real and imaginary parts of Gaussian noise and then taking its magnitude. Figure 2 shows the cross section of BrainWeb phantom corrupted by Gaussian noise with $\sigma=13 \%$ and the results obtained by proposed algorithm. Horizontal, coronal and sagittal views are shown below. 


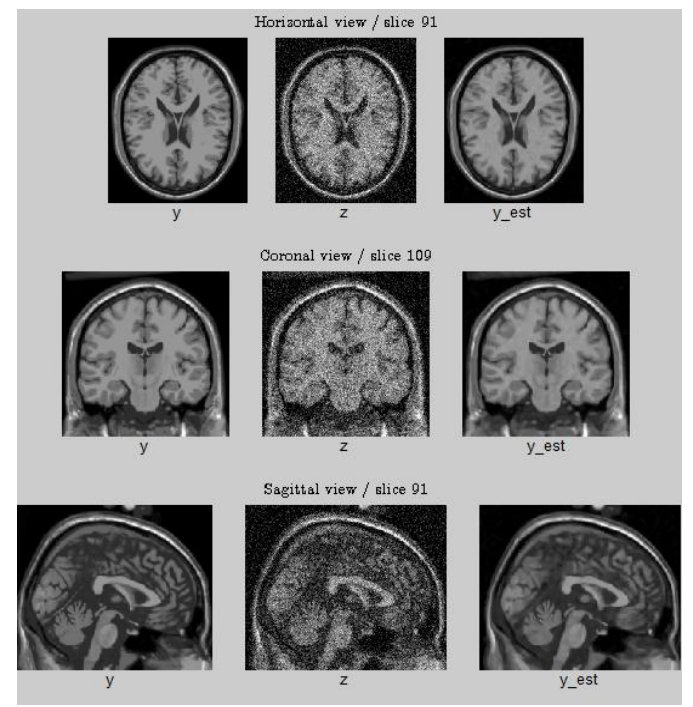

Figure. 2. Denoising results of Proposed Method

\section{CONCLUSION}

The paper has proposed a cascaded scheme: first, we have used NL-PCA method which includes PCA decomposition and hard thresholding; second, we have presented Wiener filtering stage which further refines the denoised image. Our proposed method has been compared with current state of art algorithms and shows improved performance in terms of PSNR. Experimental results demonstrate that proposed algorithm not only outperforms the other denoising algorithms but also significantly attains better visual appearance.

\section{ACKNOWLEDGMENT}

We are grateful to Dr. Alessandro Foi for helping us in implementation of BM4D algorithm. We would also like to thank J. V. Manjón for providing PRI-NL-PCA algorithm.

\section{REFERENCE}

[1] A. Buades, B. Coll, and J. M. Morel, "A Review of Image Denoising Algorithms, with a New One," Multiscale Modeling \& Simulation Multiscale Model. Simul., vol. 4, no. 2, pp. 490$530,2005$.

[2] J. Manjon, J. Carbonellcaballero, J. Lull, G. Garciamarti, L. Martibonmati, and M. Robles, "MRI denoising using Non-Local Means," Medical Image Analysis, vol. 12, no. 4, pp. 514-523, 2008.

[3] P. Coupé, P. Yger, and C. Barillot, "Fast Non Local Means Denoising for 3D MR Images," Medical Image Computing and Computer-Assisted Intervention - MICCAI 2006 Lecture Notes in Computer Science, pp. 33-40, 2006.

[4] P. Coupe, P. Yger, S. Prima, P. Hellier, C. Kervrann, and C. Barillot, "An Optimized Blockwise Nonlocal Means Denoising Filter for 3-D Magnetic Resonance Images," IEEE Transactions on Medical Imaging IEEE Trans. Med. Imaging, vol. 27, no. 4, pp. 425-441, 2008.

[5] P. Coupé, P. Hellier, S. Prima, C. Kervrann, and C. Barillot, "3D wavelet subbands mixing for image denoising,” J. Biomed. Imag., vol. 2008, pp.1-11, Jan. 2008. 
[6] Y. Gal, A. Mehnert, A. Bradley, K. Mcmahon, D. Kennedy, and S. Crozier, "Denoising of Dynamic Contrast-Enhanced MR Images Using Dynamic Nonlocal Means," IEEE Transactions on Medical Imaging IEEE Trans. Med. Imaging, vol. 29, no. 2, pp. 302-310, 2010.

[7] H. Liu, C. Yang, N. Pan, E. Song, and R. Green, "Denoising 3D MR images by the enhanced non-local means filter for Rician noise," Magnetic Resonance Imaging, vol. 28, no. 10, pp. $1485-1496,2010$.

[8] J. Hu, Y. Pu, X. Wu, Y. Zhang, and J. Zhou, "Improved DCT-Based Nonlocal Means Filter for MR Images Denoising," Computational and Mathematical Methods in Medicine, vol. 2012, pp. 1-14, 2012.

[9] J. V. Manjón, P. Coupé, A. Buades, D. L. Collins, and M. Robles, "New methods for MRI denoising based on sparseness and self-similarity," Medical Image Analysis, vol. 16, no. 1, pp. $18-27,2012$.

[10] M. Maggioni, V. Katkovnik, K. Egiazarian, and A. Foi, "Nonlocal Transform-Domain Filter for Volumetric Data Denoising and Reconstruction," IEEE Transactions on Image Processing IEEE Trans. on Image Process., vol. 22, no. 1, pp. 119-133, 2013.

[11] J. V. Manjón, P. Coupé, and A. Buades, "MRI noise estimation and denoising using nonlocal PCA," Medical Image Analysis, vol. 22, no. 1, pp. 35-47, 2015.

[12] P. Coupé, M. Munz, J. V. Manjón, E. S. Ruthazer, and D. L. Collins, "A CANDLE for a deeper in vivo insight," Medical Image Analysis, vol. 16, no. 4, pp. 849-864, 2012.

[13] D. S. Marcus, T. H. Wang, J. Parker, J. G. Csernansky, J. C. Morris, and R. L. Buckner, "Open access series of imaging studies (OASIS): Crosssectional MRI data in young, middle aged, nondemented, and demented older adults," J. Cognit. Neurosci., vol. 22, no. 12, pp. 26772684, 2010. 